Document downloaded from:

http://hdl.handle.net/10251/37057

This paper must be cited as:

Rodríguez Villalobos, A.; Ruiz García, R. (2012). A study on the effect of the asymmetry on real capacitated vehicle routing problems. Computers and Operations Research. 39(9):2142-2151. doi:10.1016/j.cor.2011.10.023.

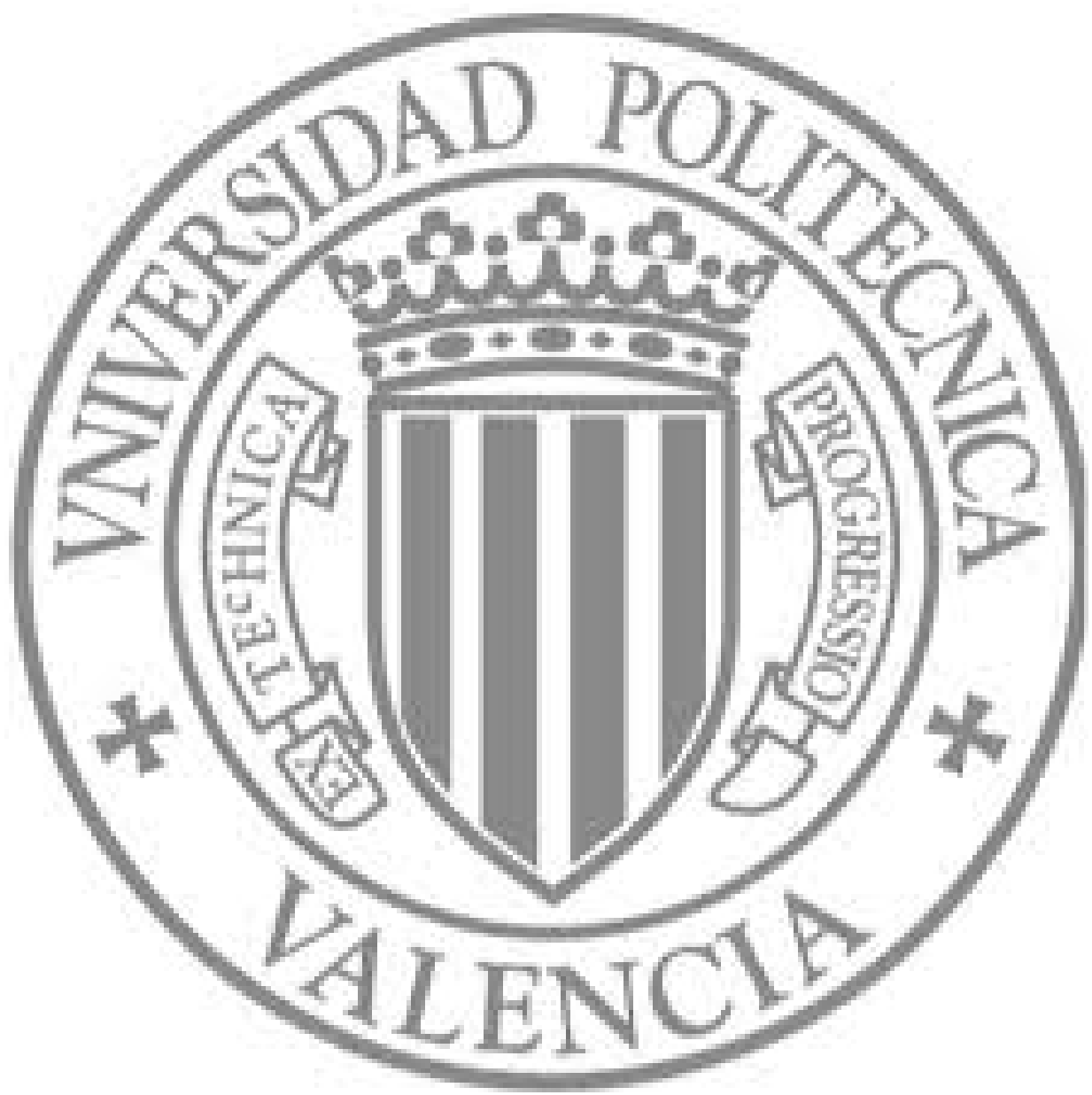

The final publication is available at

http://dx.doi.org/10.1016/j.cor.2011.10.023

Copyright Elsevier 


\title{
A study on the effect of the asymmetry on real capacitated vehicle routing problems
}

\author{
Alejandro Rodríguez ${ }^{1 *}$ Rubén Ruiz ${ }^{2}$ \\ ${ }^{1}$ Grupo de Sistemas de Optimización Aplicada, Instituto Tecnológico de Informática, \\ Universitat Politècnica de València, Pza. Ferrándiz Carbonell 2, 03801 Alcoy, Spain. \\ arodriguez@doe.upv.es \\ ${ }^{2}$ Grupo de Sistemas de Optimización Aplicada, Instituto Tecnológico de Informática, \\ Universitat Politècnica de València, Camino de Vera s/n, 46021 Valencia, Spain. \\ rruiz@eio.upv.es
}

October 26, 2011

\begin{abstract}
Matrices with distances between pairs of locations are essential for solving vehicle routing problems like the Capacitated Vehicle Routing Problem $(C V R P)$, Traveling Salesman Problem (TSP) and others. This work deals with the complex reality of transportation networks and asymmetry. Through a series of comprehensive and thorough computational and statistical experiments we study the effect that many factors like asymmetry, geographical location of the depot and clients, demand, territory and maximum vehicle capacity have in the solution of $C V R P$ instances. We examine both classical heuristics as well as current state-of-the-art metaheuristics and show that these methods are seriously affected by the studied factors from a solution time and quality of solutions perspective. We systematically compare the solutions obtained in the symmetric scenario with those obtained in the real asymmetric case at a quantitative as well as a qualitative level, with the objective of carefully measuring and understanding the differences between both cases.
\end{abstract}

Keywords: Asymmetry, Capacitated Vehicle Routing Problem, Algorithms, Road Transportation Networks

\footnotetext{
${ }^{*}$ Corresponding author. Tel/Fax: +34966528489
} 


\section{Introduction}

In many vehicle routing problems and also in several other aspects of logistics, a distance matrix between pairs of locations is needed. This matrix is needed for the Traveling Salesman Problem (TSP) (Flood, 1956), Capacitated Vehicle Routing Problem $(C V R P)$ (Clarke and Wright, 1964), and for almost any other variant of routing problem (Toth and Vigo, 2001). In particular, in the $C V R P$ each client or location $j$ from the location set $V$ is associated with a given demand quantity or requirement $d_{j}$ that is known in advance, non-negative and deterministic. The demand models a requirement of $d_{j}$ units of product by client $j$ that has to be delivered by a single truck, i.e., without splitting the demand into various trucks. A fleet $K$ of homogeneous vehicles is available, each one of them with a given capacity of service $C$ which obviously has to be higher than all demand $d_{j}, \forall j \in V$. The fleet is stationed at a central depot, usually referred to as node 0 (or node 1 )with no demand. All clients must be served by exactly one vehicle. This classic problem entails no further constraints apart from the capacity of all vehicles, which cannot be exceeded. The objective in the $C V R P$ is to obtain a set of routes, usually one per vehicle, so that the total cost, measured as the total distance traveled by all vehicles,

is minimized. Other objectives could be to minimize travel time, costs, etc. (Laporte, 2007).

Given an instance of the $C V R P$ with $n$ locations or nodes, the distance matrix between node pairs $o, d$, where $o, d \in V, o \neq d$, is denoted by $C_{[n \times n]}$. The diagonal of this matrix contains zeros and is usually disregarded. As a result, the matrix contains $n \times(n-1)$ elements with all the distances between nodes.

In the vast majority of the routing literature (Laporte, 2009), locations or nodes are determined by simple coordinates on the $2 \mathrm{D}$ plane and the distances are calculated by the Euclidean formula. A more elaborate calculation can be done by taking the geographical locations of the nodes in the terrestrial surface (geolocations in latitude and longitude) and measuring the orthodromic distance between them using the great-circle distance formula of Vincenty (1975). In both cases, the distances between nodes $O$ and $d$ is the same as the distance between $d$ and $o$, i.e., $c_{o d}=c_{d o}, \forall o, d \in V, o \neq d$. As a result, matrix $C$ is symmetric and can be reduced to either the upper or lower triangular with $\frac{n \times(n-1)}{2}$ elements. Symmetric matrices result in symmetric problems where the orthodromic arc or distance $\left(c_{o d}=c_{d o}\right)$ in many cases a weak lower bound of the real shortest route between two locations when this route is calculated considering transportation networks. When one takes into account the intricate reality of transportation networks with roads, traffic circles, streets, one way streets, etc., the distance matrix is inevitably more complex and 
asymmetric where generally, $c_{o d} \neq c_{d o}$ and distances are usually larger than symmetric Euclidean distances (Laporte et al., 1986).

In the 1970s, some authors set about estimating real distances (which were, at the time, challenging to obtain as the Geographical Information Systems or GIS were yet to be developed) by transforming orthodromic calculations (Christofides and Eilon, 1969). Other authors developed mathematical functions in order to approximate real distances like Love and Morris (1972). After the work of Daganzo (1984), Love and Morris (1988) and Dubois and Semet (1995) it is concluded that such approximations are not practical for daily and operational use by companies. Furthermore, and most importantly, the degree of asymmetry in the distance matrix cannot be easily estimated as it greatly depends on various factors (Daganzo, 1984). Nodes separated by large distances usually result in more symmetric matrices as long distance transportation entails using two-way highways. However, nodes located inside cities, and particularly cities with old historic centers, result in highly asymmetric distance matrices.

Despite this reality, there is a widespread usage of Euclidean matrices, mainly motivated by the difficulty and cost of obtaining real distance matrices. Calculating real distances requires a big computational effort as $n \times(n-1)$ shortest paths between nodes have to be obtained. Each shortest path is a complex calculation comprising of hundreds of roads, turns, etc. In a problem with a thousand nodes, almost a million shortest paths are needed. While the computational cost is still large, fortunately since the 1990s researchers have had powerful GIS systems (such as Google Maps) at their disposal (Goodchild and Kemp, 1990). Together with geo-spatial databases and their Advanced Programming Interfaces (APIs), calculating real distance matrices is today a reality.

This piece of research studies the $C V R P$ from a very different perspective. A sizeable chunk of the literature has focused on developing efficient algorithms and methods for optimizing the symmetric and Euclidean $C V R P$. This paper follows a different direction as we set out to study the effect that the asymmetry of the distance matrix, as well as many other factors, have over the effectiveness and efficiency of relevant heuristic and metaheuristic methods for the $C V R P$. We closely examine how the quality of the solutions and the quantity of CPU time employed by the studied methods is affected. We also compare the solutions obtained using symmetric and asymmetric matrices, in order to ascertain how different these solutions are and to what extent using Euclidean matrices is acceptable for real environments.

This work is a natural extension of a previous study by the same authors on the effect of the asymmetric and other factors over heuristic methods for the much simpler Traveling Salesman Problem (TSP) (Rodríguez and Ruiz, 2011). One objective of the present pa- 
per is to corroborate if the conclusions obtained in the previous study also apply to more complex routing problems. In order to do so, we will carry out a comprehensive study with different transportation networks, locations of clients and depots, problem sizes, asymmetry degrees, levels of demand and transport capacity, solution methods and CPU time. The rest of the paper is organized as follows: Section 2 presents the research hypotheses and questions, as well as a more formal definition of the studied factors, variables and the experimental design. Section 3 summarizes the main obtained results as regards computational time, quality of solutions and a comparison of solutions quantitatively and qualitatively. Lastly, Section 4 concludes the paper.

\section{Impact of the asymmetry and other factors}

The following hypotheses are studied in this paper: Asymmetry affects the effectiveness and efficiency of $C V R P$ heuristics and metaheuristics. The geographical location of clients and the central depot in the territory translates into different degrees of asymmetry in the distance matrix and in turn affects the effectiveness and efficiency of the studied methods. The asymmetry of the transportation network has a specific effect over those methods based on the planar Euclidean geometry. Furthermore, the following research questions are raised: To what extent are the solutions obtained in symmetric and asymmetric scenarios similar? What is the result of taking the symmetric solution (solution calculated with a symmetric distance matrix) and calculating it with the real asymmetric distance matrix and vice versa?

In order to either confirm or to refute the previous hypotheses and to answer the posed questions we carry out a comprehensive computational and statistical testing campaign with a set of $C V R P$ instances, whose details are explained next.

\subsection{Studied factors}

Territory: It is the real geographical region where the clients and central depot are located. A boundary is created by two opposed geographical coordinates (latitude and longitude) that induce a quadrant. The region of choice, related to the Iberian peninsula, is studied at three territory variants, namely short, medium and large distance. In the short distance territory routes have to deal with urban transportation (mostly Madrid and its surroundings) Medium distance includes routes in cities plus longer routes that communicate other cities through highways. Lastly, large distance deals with a large national territory and includes heavy usage of highways. More details about this factor, 


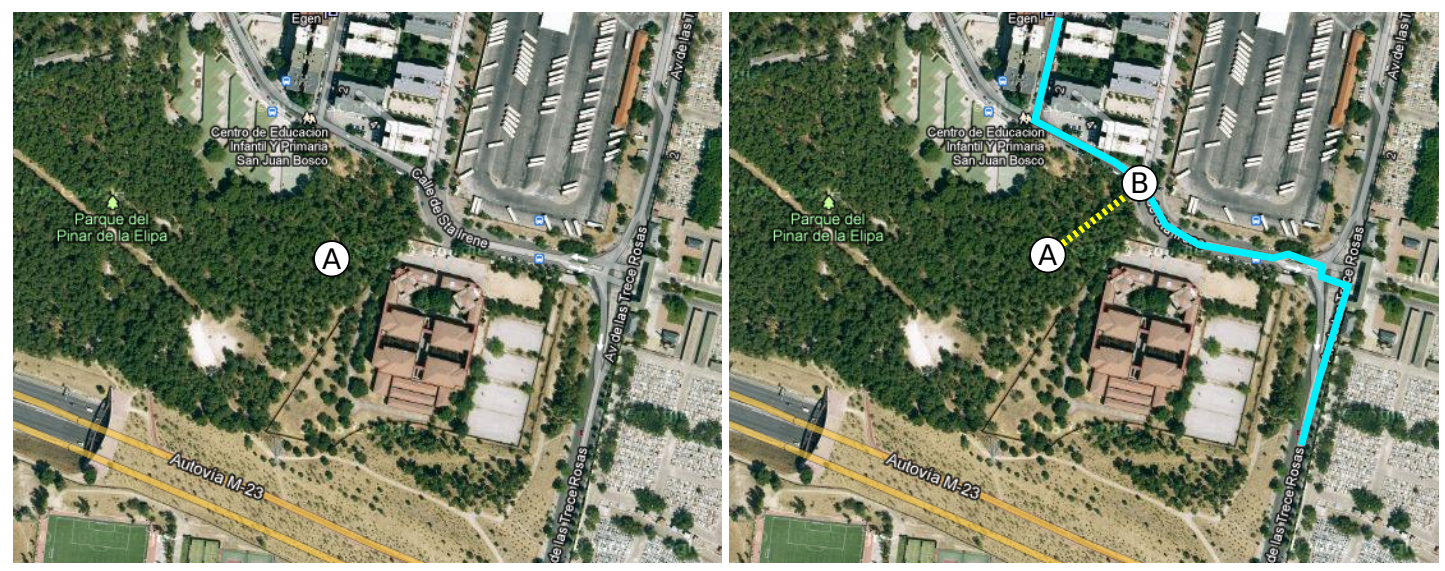

Figure 1: Location correction according to the transportation network. Left picture: a node placed in an innaccesible location (A). Right picture: final corrected location (B).

including longer explanations and graphical examples are given in Rodríguez and Ruiz (2011).

Location: This factor determines how the clients or nodes are distributed inside the territory. Three variants are studied: random placement, grid placement and radial placement. This factor was also studied previously and all details are given in Rodríguez and Ruiz (2011). In all cases, including the random variants, all nodes (and depot) are located in accessible places inside the transportation network. Furthermore, we also make sure that a path exists between each node and all others. This is further explained by means of the following example, supported by Figure 1; Let us suppose that, when building the instance and after a random placement of nodes, a node (A) has been placed in a geographically inaccessible place. Inaccessible places are, for example, and as depicted in the Figure, a forest. Other examples could be in the middle of a field, or even inside of a block of apartments. We use a GIS function which basically moves the client to the nearest accessible location, shown on the right picture in Figure 1. This movement normally is within a few meters from the original inaccessible location. Note that this final location could perfectly correspond to a real client.

Number of nodes: This factor is just the number of clients in a given territory. It is a quantitative factor with 10 levels $n=\{50,100,150, \ldots, 500\}$. Note that this is the last factor employed in this paper that was also studied as it is by Rodríguez and Ruiz (2011).

Depot location: This is a nominal qualitative factor that studies the effect of the placement of the depot respective to the territory and clients. We have defined three variants: Random: The depot is chosen randomly among the $n$ nodes. Centered: It is the node which is closest to the orthodromic center of the territory. Peripheral: The depot is 
the node which is farthest away from the orthodromic center and closest to the boundaries of the territory.

Symmetry: From the territory and node locations we calculate the distance matrices. In Rodríguez and Ruiz (2011), the authors calculated these matrices in five different ways. However, only two of them are of interest for the $C V R P$. As a result we only have two variants for this nominal factor: Orthodromic: A symmetric distances matrix obtained by calculating the orthodromic distances between pairs of nodes using their geolocations. Asymmetric: real distances matrix using a GIS (Google Maps) and calculating the shortest route between any two clients, similarly as any turn-by-turn navigation system would do.

Demand and maximum capacity (DemCap): Service demand $d_{j}$ of each client $j$ and the maximum transport capacity $C$ of the trucks are so intimately related that they are considered as a single factor in this study. Service demand is modeled as a two level quantitative factor where demand $d_{j}$ is sampled from a uniform random distribution in two intervals: Small $(P)$ using $U[1,10]$ and Large $(L)$ using $U[50,100]$ as demand units, respectively. Usually, large distance transportation requires relatively big demands served by big trucks, usually of three axels. Conversely, short distance comprises the delivery of demands in greater number but smaller volume and with smaller trucks. This is translated into longer routes for large distance with a smaller number of stops during the route $(s)$ and relatively shorter routes in short distance transportation but with a higher number of stops $s$. In order to model this situation and to calculate the maximum vehicle capacity $C$, we employ a number of stops generator $G(p)$ defined in equation (1) with a probability following a triangular distribution as a function of a parameter $p \in U[0,1]$. Table Table 1 shows the selected values $a, b, c$ of the triangular distribution related to $G(p)$ according to the territory factor.

$$
G(p)= \begin{cases}a+\sqrt{p(b-a)(c-a)} & p \leq \frac{c-a}{b-a} \\ b-\sqrt{(1-p)(b-a)(b-c)} & p>\frac{c-a}{b-a}\end{cases}
$$

\begin{tabular}{llll}
\hline Territory & Minimum (a) & Maximum (b) & Mode (c) \\
\hline Short distance & 10 & 25 & 25 \\
Medium distance & 10 & 25 & 17.5 \\
Large distance & 10 & 25 & 10 \\
\hline
\end{tabular}

Table 1: Number of stops $s$ according to the territory and triangular distribution.

From $G(p)$ and the average service demand $\bar{d}_{j}$ of all clients $j \in V$ we define the maximum capacity $C$ as in equation (2): 


$$
C=\max d_{j}+(G(p)-1) \bar{d}_{j} \quad \forall j \in V
$$

In the set of proposed instances, service demand $d_{j}$, the related vehicle capacity $C$ and the number of stops $s$ are rounded to the nearest integer. It is important to note that the number of stops $s$, number of nodes $n$ and the number of routes $k$ will be in the interval $\{n=50, k=5, s=10, \ldots, n=500, k=20, s=25\}$. This is in concordance with what can be found in the most common benchmarks from the literature like those of Christofides and Eilon (1969), Golden et al. (1977), TSPLIB1, Taillard (1993), and Fisher (1994) among others; where completely random instances and some real cases from industries are used.

\subsection{Experimental design and response variables}

The six studied factors, along with the studied levels and variants are summarized in Table 2. Note that the last row shows the total number of levels or variants for each factor and that the service demand and vehicle capacity is considered as a single factor "DemCap".

\begin{tabular}{llllll}
\hline Territory $(\mathbf{T})$ & Location $(\mathbf{L})$ & Symmetry $(\mathbf{M})$ & Number of nodes $(n)$ & Depot location & DemCap \\
\hline Short distance & Random & Orthodromic & 50 & Random & Small \\
Medium distance & Grid & Asymmetric & 100 & Centered & Large \\
Large distance & Radial & & Peripheral & \\
& & & 500 & 3 & 2 \\
\hline 3 & 3 & 2 & 10 & 3 & \\
\hline
\end{tabular}

Table 2: Factors and their corresponding studied levels or variants.

In this paper we employ a full factorial experimental design. All combinations of the levels and variants of the factors are studied which results in $3 \times 3 \times 2 \times 10 \times 3 \times 2=$ 1, 080 treatments. For each treatment, five different random instances are generated which produces a grand total of 5,400 CVRP instances. The full factorial design allows for the study of the effects of each factor as well as the interactions of any level over the following response variables:

Deviation from best known solution $\Delta S_{i}^{*}$ : According to equation (3) it is the percentage relative deviation of the total distance traveled by the vehicles in the solution obtained with algorithm $A$ for instance $i\left(S_{i, A}\right)$ with respect to the best solution known for that very same instance $\left(S_{i}^{*}\right)$.

\footnotetext{
${ }^{1}$ http://comopt.ifi.uni-heidelberg.de/software/TSPLIB95/
} 


$$
\Delta S_{i}^{*}=\frac{S_{i, A}-S_{i}^{*}}{S_{i}^{*}} \cdot 100
$$

Success rate: Calculated as the number of times a given algorithm attains the best known solution.

CPU time: For each instance $i$ it is the real CPU time employed by a given algorithm to obtain a solution. It does not consider input/output operations or other operating system times as recommended by Alba (2006). For stochastic algorithms in which every instance is run several times, this CPU time will be an average of all runs.

Finally, a very important studied factor, not related with the instances, is the algorithm. We have selected the following $C V R P$ methods:

- Algorithm of Clarke and Wright (1964) (CW). It is a very well known and thoroughly studied heuristic that will serve as a baseline indicator of performance.

- Sweep algorithm by Gillett and Miller (1974) (SW). Another simple well known constructive method that works over two phases and that is strongly based in the planar properties of the 2D plane for the first phase. For the second phase, our implementation uses the well known efficient implementation of the Lin-Kernighan heuristic by Helsgaun (2000) which is currently considered among the highest performing heuristics for the TSP. For this later heuristic we employed the following parameters: recommended parameters by the author.

- General heuristic of Pisinger and Røpke (2007) (PR). It is a unified heuristic that works for several variants of routing problems and that uses an Adaptive Large Neighborhood Search (ALNS). It is a very capable and robust method. Parameters: recommendations from the author according to the original paper for both compilation and execution.

- Memetic algorithm of Nagata (2007) (NA). Similar to PR, NA is a very powerful and recent $C V R P$ metaheuristic. Parameters: recommendations from the author according to the original paper; 10 trials, population size $=100,30$ children, 2 parents.

The previous algorithms have been selected by their performance and recognition. We have strived for a balance between simple classical techniques and current and state-ofthe-art methods. Algorithms NA and PR were run from the original code which was kindly provided by their respective authors. No code modification was carried out and the methods were run according to their recommendations. Initially, we set no time limit 
on computational times, i.e., algorithms have a stopping criterion set by their original authors. The metaheuristics PR and NA are stochastic and therefore, five different runs are carried out for each instance. In total, there are 64,800 computational results.

\subsection{Computational setting}

All experiments are run on a cluster of 30 blade severs, each one containing two Intel XEON 5254 processors running at $2.5 \mathrm{GHz}$ with $16 \mathrm{~GB}$ of RAM memory. Each cluster has two processors with four cores each ( 8 cores per cluster) but experiments are carried out in virtualized Windows XP machines, each one with one virtualized processor and 2 GB of RAM memory. For the generation and calculation of the 2,700 matrices, (symmetric and assymetric) we needed a single blade equivalent wall time of 4,708 hours, almost solely employed for the calculation of the asymmetric matrices as the symmetric distances are almost instantaneously calculated. The single blade equivalent wall time needed for obtaining the results of 64, 800 experiments was of 12,704 (almost 530 days!). As a result, a possible contribution of this paper is in the form of a freely available benchmark of real asymmetric $C V R P$ instances (more than 2.15 GBytes of data) which can be seen as complementary to existing and well recognized benchmarks. The instances are available at http://soa.iti.es/problem-instances.

\section{$3 \quad$ Experimental results}

After running all experiments, and in order to understand the effect of the asymmetry and all other factors over the response variables, several multifactor Analyses of Variance (ANOVA) were carried out. ANOVA is a parametric statistical model. As such, there are three main hypotheses that must be met, all of them require the residuals from the experiment to follow a normal distribution, to be homocesdastic and to be independent (not self correlated). In an experiment with such a large dataset, such hypotheses are easily met and we did not observe any serious deviation.

\section{$3.1 \quad$ CPU time}

Some of the most interesting conclusions come after analyzing the CPU time response variable. After all, one could think that, apart from the number of nodes $n$, no other studied factor should have a large effect on the CPU time that a given algorithm needs to solve an instance. Recall that no CPU time limit was established for all methods. We are working with a significance level of $\alpha=0.05$. The resulting multifactor ANOVA produced 
27 statistically significant relations, including single factors and two way interactions. Table 3 shows a summary of the ANOVA table, including the $p$-values. Due to limitations on space, only single factor effects are shown.

\begin{tabular}{llllll}
\hline Source & $\begin{array}{l}\text { Sum of } \\
\text { squares }\end{array}$ & $\begin{array}{l}\text { Degrees } \\
\text { of freedom }\end{array}$ & $\begin{array}{l}\text { Mean } \\
\text { square }\end{array}$ & $F$-Ratio & $p$-value \\
\hline Main effects & & & & & \\
A:DemCap & $1.934 E 8$ & 1 & $1.934 E 8$ & $1,519.73$ & 0.0000 \\
B:Depot location & $4.385 E 7$ & 2 & $2.192 E 7$ & 172.24 & 0.0000 \\
C:Territory (T) & $9.311 E 6$ & 2 & $4.655 E 6$ & 36.57 & 0.0000 \\
D:Location (L) & $2.052 E 7$ & 2 & $1.026 E 7$ & 80.61 & 0.0000 \\
E:Symmetry (M) & 617,811 & 1 & 617,811 & 4.85 & 0.0276 \\
F:Number of nodes $(n)$ & $4.305 E 9$ & 9 & $4.784 E 8$ & $3,757.57$ & 0.0000 \\
G:Algorithm & $2.893 E 10$ & 3 & $9.645 E 9$ & $75,756.96$ & 0.0000 \\
Residual & $8.228 E 9$ & 64,631 & 127,319 & & \\
Total (corrected) & $8.590 E 10$ & 64,799 & & & \\
\hline
\end{tabular}

Table 3: Results of the analysis of variance for CPU time response variable.

As we can see, the strongest effect (larger F-Ratios, which are the quotient between the variance generated by the different levels of the factor and the residual variance, calculated as a quotient between the mean squares) is due to the factors Algorithm and number of nodes $(n)$. These two are obvious factors. Quick heuristics like CW and SW are almost instantaneous while complex metaheuristics like PR and NA are much more time consuming. The same can be said about $n$, i.e., the larger the problem, the longer the CPU time. What is of interest is how these two strong factors interact with all other factors and the behavior of other factors themselves. For example, the Location (L) factor strongly affects CPU time $(F$-Ratio $=80.61, p$-value $=0.00)$. Furthermore, this factor affects the NA algorithm much more than all other studied methods as CPU time increases a $15 \%$ in the grid and radial locations with respect to random locations. Comparatively, PR's CPU time are barely affected by this factor, as shown in Table 4. Also of interest is the simple CW heuristic, whose CPU times, albeit small, increase by more than $34 \%$ for the radial locations with respect to random.

\begin{tabular}{lllll}
\hline Algorithm & Random & Grid & Radial & average \\
\hline CW & 2.17 & 2.56 & 2.91 & 2.55 \\
NA & $1,351.49$ & $1,552.47$ & $1,572.91$ & $1,492.29$ \\
PR & 207.46 & 198.20 & 196.99 & 200.88 \\
SW & 1.27 & 1.24 & 1.28 & 1.26 \\
\hline average & 649.85 & 729.77 & 737.81 & \\
\hline
\end{tabular}

Table 4: Average CPU time as a function of the Algorithm and Location (L) factors. 


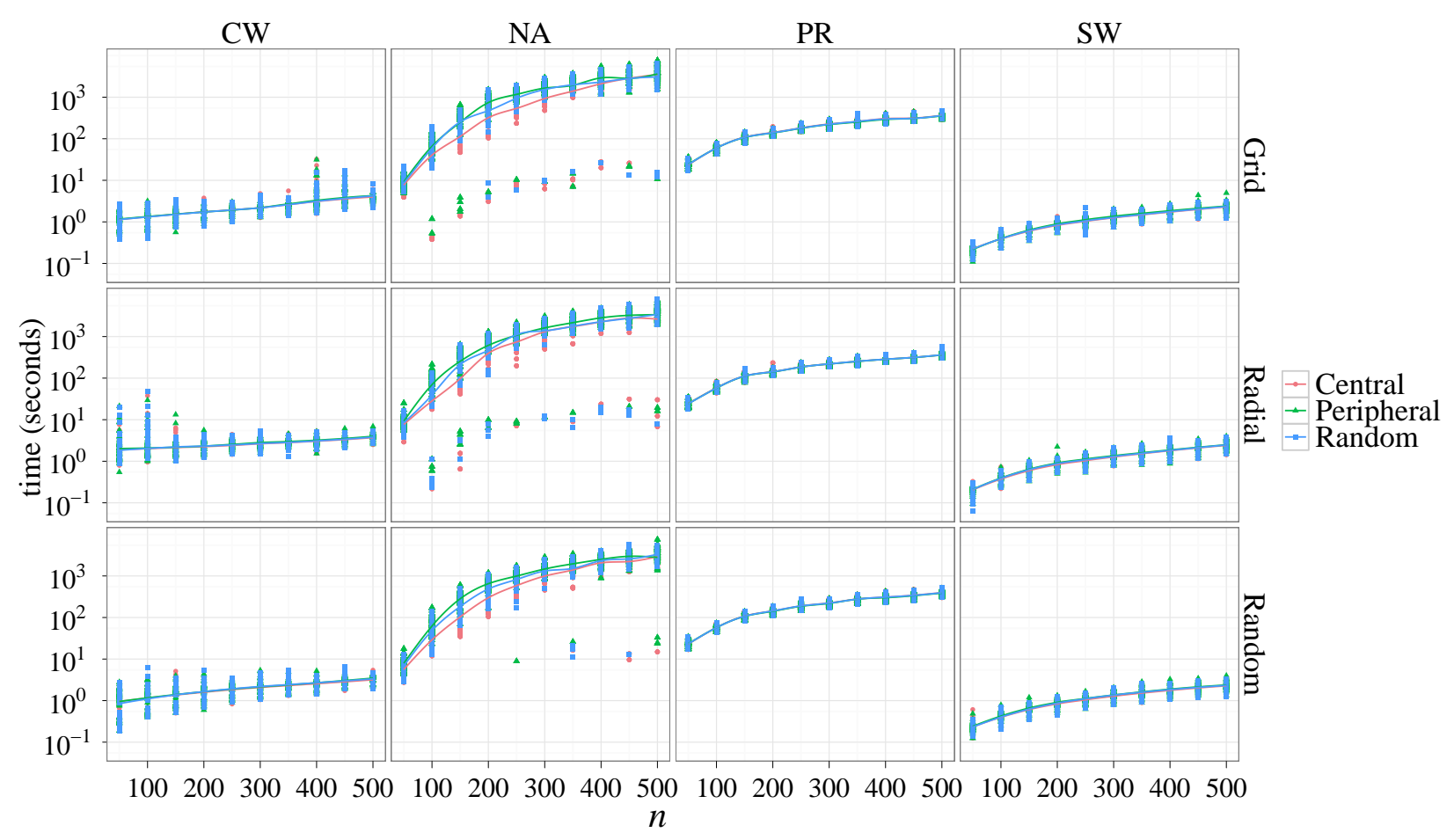

Figure 2: CPU time for the combinations of Algorithm, Location (L), Depot location and Number of nodes $(n)$.

Figure 2 depicts a series of scatter plots for all combinations of algorithms and node locations. The $\mathrm{x}$-axis shows the number of nodes $n$ and the $\mathrm{y}$-axis the CPU time in seconds in a logarithmic scale. Different colors and symbols are used for the Depot location factor. From the ANOVA it can be observed that the Depot location has a significant effect on the CPU time $(F$-Ratio $=172.24, p$-value $=0.00)$, especially for the NA method. When the depot is located peripherically, CPU times increase by $22 \%$ with respect to a centrally located depot. Once again PR is robust with respect to the depot location with a meager $2 \%$ increase in CPU time from the central to the peripheral. Both $\mathrm{CW}$ and SW increase their CPU times by about $5 \%$ with a peripherically located depot with respect to a central depot. The location of the nodes (factor L) also affects CPU time in a significant way. According to our results, this effect is more pronounced with the NA method (an increase of CPU time of about $15 \%$ in Grid and Radial with respect to Random location). PR is again robust regarding this factor. In relation to the number of nodes $n$, our experiments confirm the direct and exponential effect that the size of the problem has on CPU time. This effect is observed in all cases both for symmetric as well as for asymmetric cases.

Territory has a small, but, definitely statistically significant effect over CPU time 
$(F$-Ratio $=36.57)$. This result was already observed in our previous work for the TSP (Rodríguez and Ruiz, 2011). Large distance territories have less asymmetric matrices and less variability between nodes, which results in longer CPU times for the methods. This effect is observed for the algorithm CW and Large distances where CPU times are increased by $68 \%$ when compared with the times obtained for the Short distance territory (with more variability and asymmetry in the distances, which helps when searching for good solutions quickly).

A strong factor specific to the $C V R P$ is the DemCap factor which, as we recall, it models the relationship between the service demand and vehicle capacity $(F$-Ratio= 1,519.73, $p$-value $=0.00)$. For Small DemCap the average CPU time is increased by $40 \%$ for the whole dataset. A possible interpretation of these results, when relating DemCap, Territory and Asymmetry, is that for Short distance and Small DemCap the complexity of the problem increases and CPU times worsen when compared to Medium-Large distances and Large DemCap. In other words, the higher the number of stops per vehicle, the higher the complexity and hence, CPU time needed. Furthermore, if the Territory is Short distance, the CPU times increase even further. However, if each vehicle has less stops and has to travel Medium-Large distances, the problem ends up easier to solve.

Our results point to the idea that the different degree of asymmetry in the transportation networks clearly and statistically affect the CPU time $(p$-value $=0.02)$. As detailed in Table 5, while for NA and PR the CPU times increase by a measly $2 \%$, simple algorithms increase CPU times by as much as $15 \%$.

\begin{tabular}{llll}
\hline Algorithm & Asymmetric & Orthodromic & average \\
\hline CW & 2.42 & 2.67 & 2.55 \\
NA & $1,507.47$ & $1,477.12$ & $1,492.29$ \\
PR & 202.57 & 199.19 & 200.88 \\
SW & 1.09 & 1.43 & 1.26 \\
\hline average & 712.81 & 698.81 & \\
\hline
\end{tabular}

Table 5: Average CPU time as a function of the Algorithm and Symmetry (M) factors.

We finally provide a means plot with confidence intervals. We employ the most restrictive technique for calculating the confidence intervals around the means: the Tukey's Honest Significant Difference (HSD) intervals. As shown in Figure 3, the means and their corresponding Tukey's HSD intervals at a 95\% confidence level for the Symmetry (M) factor do not overlap. Therefore, the difference is statistically significant. 


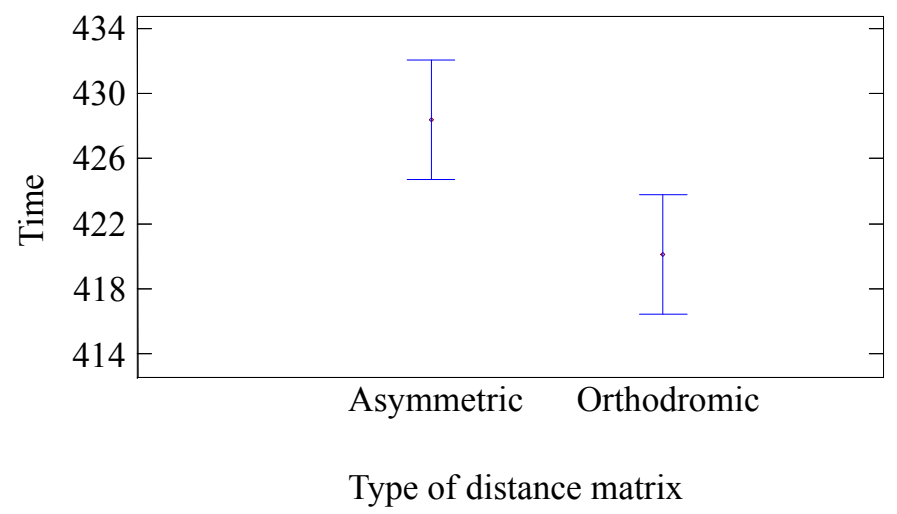

Figure 3: Means plot for CPU time with Tukey's Honest Significant Difference (HSD) 95\% confidence intervals for the Symmetry (M) factor.

\subsection{Quantitative and qualitative comparison}

In order to observe the differences between the symmetric and asymmetric solutions we employ some indicators. These are based on the following data:

$S O$ : Is the objective value (in our case total traveled distance) after taking the routes obtained by an algorithm using the symmetric distance matrix.

$S A$ : Is the total traveled distance after taking the routes obtained by an algorithm using an asymmetric distance matrix.

$S O_{A}$ : Total traveled distance of the routes obtained by an algorithm using the symmetric distance matrix but recalculated using the real asymmetric matrix, i.e., the algorithm works with a symmetric matrix but after the solution has been obtained it is recalculated with the real distance matrix.

$S A_{O}$ : Is the opposite of $S O_{A}$. Take a solution obtained with an asymmetric matrix and recalculate the total distance with the orthodromic distances.

The indicators are therefore the following:

$I_{1}$ percentage increase of $S A$ with respect to $S O$ :

$$
I_{1}=\frac{S A-S O}{S O} \cdot 100
$$

$I_{2}$ percentage increase of $S O_{A}$ with respect to $S A$. It can be negative.

$I_{3}$ percentage increase of $S A_{O}$ with respect to $S O$. It can also be negative.

In the following we further detail the previous indicators by means of a simple example. We show below the distance matrices between the depot and four clients: $O$ contains the symmetric orthodromic distances and $A$ the real distances, all measured in kilometers. 


$$
O=\left(\begin{array}{lllll}
0.000 & 2.218 & 2.545 & 1.305 & 2.585 \\
2.218 & 0.000 & 4.503 & 3.193 & 2.682 \\
2.544 & 4.503 & 0.000 & 1.313 & 3.105 \\
1.305 & 3.193 & 1.313 & 0.000 & 2.255 \\
2.585 & 2.682 & 3.105 & 2.255 & 0.000
\end{array}\right) \quad A=\left(\begin{array}{ccccc}
0.000 & 2.788 & 3.122 & 3.193 & 3.508 \\
3.023 & 0.000 & 5.341 & 5.412 & 3.722 \\
3.188 & 5.684 & 0.000 & 2.124 & 4.561 \\
2.571 & 4.349 & 3.852 & 0.000 & 2.705 \\
3.742 & 4.041 & 4.592 & 3.665 & 0.000
\end{array}\right)
$$

The demands of the clients are all equal to one and the vehicles have a maximum capacity of $C=3$. We used an exact Mixed Integer Linear Programming (MILP) model in order to obtain the optimal solution, after which $S O$ and $S A$ are calculated. The results are as follows:

Symmetric: route 1 (7.485 km.) sequence $\{1,2,5,1\}$, route $2(5.162 \mathrm{~km}$.), sequence $\{1,4,3,1\}$. Total distance $S O=12.647 \mathrm{~km}$.

Asymmetric: route $1(5.811 \mathrm{~km}$.) sequence $\{1,2,1\}$, route $2(11.693 \mathrm{~km}$.$) , sequence$ $\{1,3,4,5,1\}$. Total distance $S A=17.504 \mathrm{~km}$.

We can see that for this small example, both scenarios result in two routes. However, the routes are different and the total traveled distance is much higher in the asymmetric case. Figure 4 shows a graphical representation of the solutions.
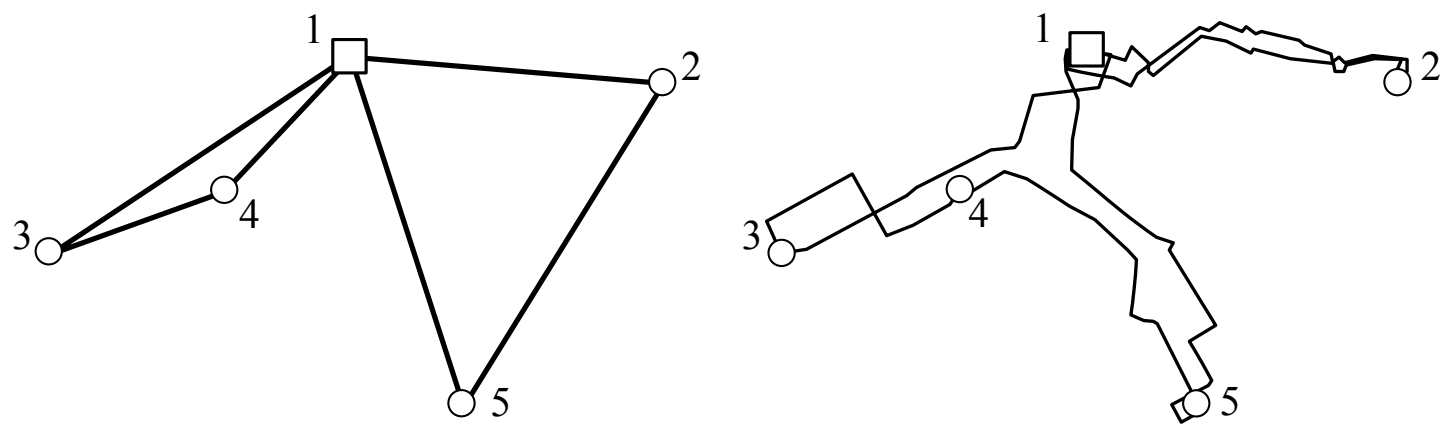

Figure 4: An example solution with symmetric routes on the left and asymmetric real routes on the right.

The indicator $I_{1}$ takes a value of $37.22 \%$ which shows the increase in the distance $S A$ with respect to $S O$. Similarly, $S O_{A}=20.485 \mathrm{~km}$. and reciprocally, $S A_{O}=13.134 \mathrm{~km}$. As a result, $I_{2}=17.03 \%$ and $I_{3}=3.85 \%$, clearly demonstrating that both solutions are far from being interchangeable. Furthermore, when closely looking at the resulting routes in both scenarios (Figure 4) we realize that the solutions are very different.

The previous $S O, S A, S O_{A}$ and $S A_{O}$ values were calculated for all 64, 800 experimental results. All three indicators were then geberated, obtaining 32, 400 results (each one 
comparing asymmetric and symmetric results). The main statistics for the three indicators are given in Table 6, One striking result is the average of $I_{1}$ with a value of $59.86 \%$, which clearly indicates that there is a large quantitative difference between the cost of the asymmetric routes with respect to the cost of the symmetric ones. Note the maximum value for $I_{1}$ of $239.35 \%$, which is more than double the total distance. Recall that the nodes are placed in the same locations, the only thing that is changing is the matrix that is being fed to the algorithms. The clear conclusion is that for almost all cases, the asymmetric and symmetric problems are indeed, two different problems.

\begin{tabular}{llll}
\hline & $I_{1}$ & $I_{2}$ & $I_{3}$ \\
\hline Average & 59.86 & 10.28 & 8.38 \\
Standard deviation & 21.36 & 13.12 & 8.44 \\
Minimum & 0.3 & -58.46 & -31.46 \\
Maximum & 239.35 & 71.74 & 154.36 \\
\hline
\end{tabular}

Table 6: Statistics of the proposed comparison indicators.

Furthermore, $I_{2}$ and $I_{3}$ allow us to quantify what happens if we compare the symmetric solution calculated with the asymmetric matrix with the real asymmetric solution and vice versa. In other words, a $I_{2}=0$ for all instances would mean that it really does not matter if we use asymmetric matrices or not since the total traveled distance of the symmetric solution calculated with the asymmetric matrix would be the same as the asymmetric solution. Table 6 clearly shows that $I_{2}$ values are, on average, greater than $10 \%$. This means that it is simply not valid to take an algorithm that only works with symmetric matrices and then, once the routes have been obtained, "recalculate" them with the real distances. Likewise just ignoring real distances altogether and hoping that the very good routes obtained with orthodromic distances will be equally good in practice is not a viable approach. Our results attest to the fact that a bold $10 \%$ in total distance is being lost, compared to the solution obtained with real asymmetric distances. A similar conclusion is reached in the case of $I_{3}$ as $I_{3}$ has an average value of $8.38 \%$. Again the conclusion is that orthodromic solutions are not related to asymmetric solutions.

Figure 5 depicts the frequency distribution histograms for all three indicators. For $I_{1}$ the majority of the cases have values between $30 \%$ and $90 \%$. For $I_{2}$ most cases lie between $10 \%$ and $30 \%$. Lastly, for $I_{3}$ we see that most data stays between $7 \%$ and $40 \%$. All these indicators and results are in stark contrast with the narrow margins (usually less than 1\%) within which most current state-of-the-art algorithms compete in the benchmarks from the literature.

All these findings corroborate those from our previous study on the TSP. Similarly to the $T S P$ case, here the differences go beyond the presented indicators and objective function 
values. Usually, the routes obtained with asymmetric matrices have little in common with the routes obtained in the symmetric case (different number of routes, different sequences of nodes, etc.).

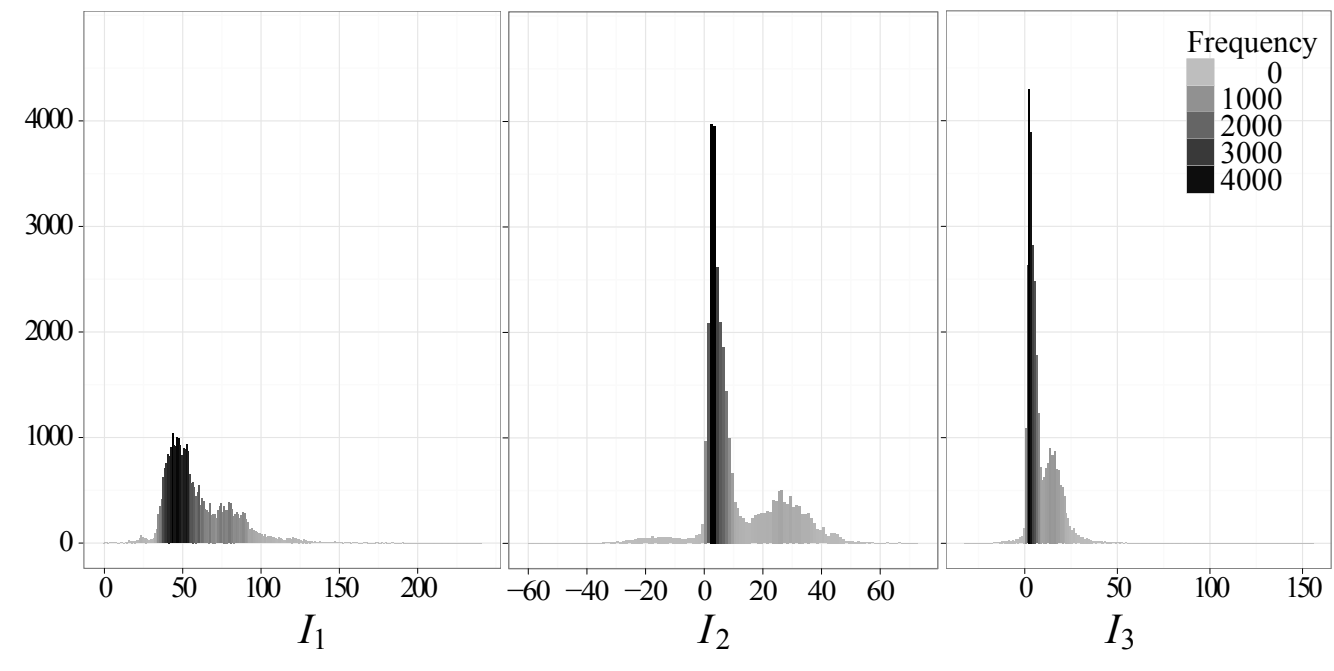

Figure 5: Frequency distribution histograms for indicators $I_{1}, I_{2}$ and $I_{3}$.

In order to better understand the relations between indicator $I_{1}$ and the other studied factors, we carried out an ANOVA. The results of the $F$-Ratios and $p$-values indicate 18 statistically significant effects: 6 factors and 12 two-way interactions. The full ANOVA table is omitted due to limitations of space. A significant effect is due to the Territory factor, $(F$-Ratio $=27355.7, p$-value $=0.00)$. For Large distance territories, the differences between the symmetric and asymmetric scenarios (as regards $I_{1}$ ) are a bit less than $44 \%$. These differences increase to $55 \%$ for Medium distance and up to $81 \%$ for Short distance. As previously mentioned, the asymmetry level increases for short distances and this is confirmed for all algorithms, as shown in Table 7 .

\begin{tabular}{lllll}
\hline Algorithm & Short distance & Medium distance & Large distance & average \\
\hline CW & 78.49 & 51.99 & 43.69 & 58.06 \\
NA & 78.93 & 51.61 & 43.52 & 58.03 \\
PR & 79.58 & 51.81 & 43.67 & 58.35 \\
SW & 103.74 & 87.68 & 43.75 & 78.39 \\
\hline average & 81.24 & 54.73 & 43.62 & \\
\hline
\end{tabular}

Table 7: Average of $I_{1}$ as a function of the Algorithm and Territory (T) factors.

Another noteworthy effect corresponds to the Depot location factor $(F$-Ratio $=3,603.5$, $p$-value $=0.00)$. As shown in Table 8 , the differences in $I_{1}$ are smaller when the depot is located in the periphery of the territory when compared to a centrally located depot 
(average of 65\%). One possible explanation is that the degree of asymmetry depends on the average distance between arcs. Since all routes start and finish at the depot, it is more likely that a centrally located depot has smaller distances to the first and last stops of every route and hence a larger asymmetry effect is observed, which increases the values of the $I_{1}$ indicator.

\begin{tabular}{lllll}
\hline Algorithm & Centered & Random & Peripheral & average \\
\hline CW & 62.32 & 59.27 & 52.58 & 58.06 \\
NA & 62.79 & 59.29 & 51.98 & 58.03 \\
PR & 63.14 & 59.63 & 52.29 & 58.35 \\
SW & 91.53 & 80.45 & 63.18 & 78.39 \\
\hline average & 65.29 & 61.19 & 53.09 & \\
\hline
\end{tabular}

Table 8: Average of $I_{1}$ as a function of the Algorithm and Depot location factors.

Lastly, the Location factor also has a significant effect over $I_{1}$. Radial locations increase $I_{1}$ with respect to Grid and Random, as shown in Table 9, This confirms the previous findings of our TSP study.

\begin{tabular}{lllll}
\hline Algorithm & Random & Grid & Radial & average \\
\hline CW & 57.02 & 55.18 & 61.97 & 58.06 \\
NA & 55.82 & 57.14 & 61.10 & 58.03 \\
PR & 56.20 & 57.37 & 61.49 & 58.35 \\
SW & 75.02 & 75.93 & 84.20 & 78.39 \\
\hline average & 57.68 & 58.64 & 63.26 & \\
\hline
\end{tabular}

Table 9: Average of $I_{1}$ as a function of the Algorithm and Location (L) factors.

To close this section we show two randomly chosen examples. The objective is to graphically show the big differences between the symmetric and asymmetric solutions. Figure [6 shows instance G-C-MR-100 on the left with 100 radially located customers in a Medium distance territory, large DemCap and a centrally located depot. Symmetric solutions are shown in blue while asymmetric routes are in red. Both solutions have five routes but this is where the similarities end. The total traveled distance in the symmetric solution is $2,149.4 \mathrm{~km}$. versus the $3,385.6 \mathrm{~km}$. of the real asymmetric solution with a $I_{1}=57.51 \%$ and notable differences in the visitation sequence of the nodes at each route. On the right of Figure 6 we have instance $\mathbf{P}-\mathbf{A}-\mathbf{C A}-\mathbf{3 5 0}$ with 350 randomly located nodes in a short distance territory with a small DemCap and a randomly placed depot. In this second example the differences are huge. The total symmetric traveled distance is 864.17 $\mathrm{km}$. which pales in comparison with the $2,140.7 \mathrm{~km}$. of the asymmetric distance and $I_{1}$ equaling $147.71 \%$. In this case, there are 17 symmetric routes and 16 asymmetric routes. 
Note that in real life, doing without one truck is, apart from the total traveled distance, a huge saving.
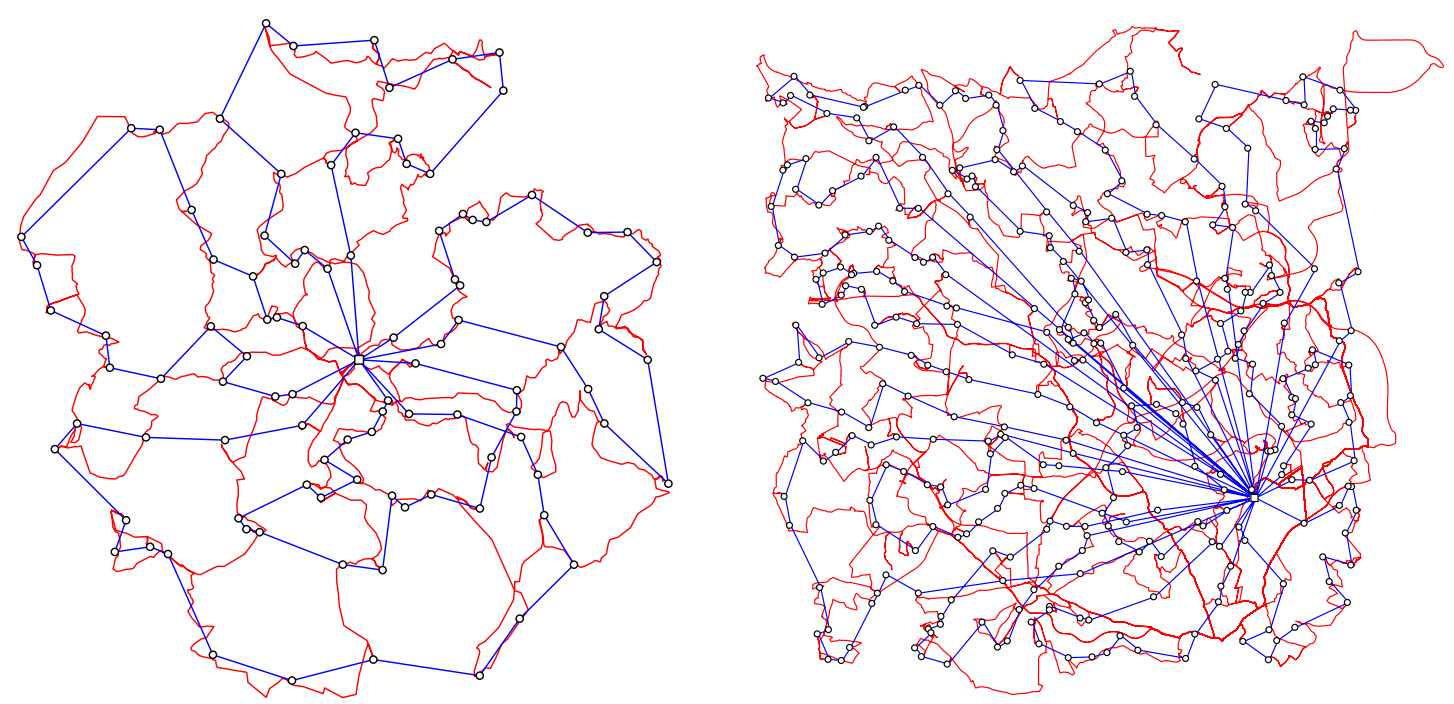

Figure 6: Symmetric solutions (in blue) and asymmetric (in red) for instances G-C-MR-100 with algorithm PR (left) and P-A-CA-350 with algorithm NA (right).

\subsection{Quality of solutions}

In previous sections we have studied that asymmetry and other studied factors have a statistically significant effect on the efficiency of the studied algorithms. However, it remains to be seen if effectiveness is affected. In our previous experiments, no CPU time limit was imposed on the different algorithms. As a result, the differences in the quality of solutions were hard to observe. Basically, algorithms were taking longer to reach comparable high quality solutions, regardless of the studied factors.

In order to study the effects on the quality of solutions we carried out additional experiments. Among the tested algorithms, NA allowed modifications in the stopping criterion. A good way of studying the effectiveness is to run NA independently for different stopping times. We define a new factor $p t$ as the percentage of allowed CPU time with respect to the original CPU time used by NA in the previous experiments where no CPU time limit was imposed $(p t=100 \%)$. The levels studied are $p t=\{10 \%, 20 \%, 40 \%, 60 \%, 80 \%, 100 \%\}$. The subset of 5, 400 results of the NA method for $p t=100 \%$ was enlarged to 32,400 results. Each instance $i$ is run from scratch, with a different random seed, for each $p t$ value in order to ensure the independence of the results and to avoid self correlation. Under this new experiment we study the response variable $p S$ according to equation (6) . A positive $p S_{i, p t}$ value indicates the deterioration in the total traveled distance for instance $i$ when 
NA is stopped at relative time $p t$ with respect to the total distance traveled when NA is run without CPU time limit $\left(p t=100 \%\right.$ or $\left.S_{i, 100}\right)$.

$$
p S_{i, p t}=\frac{S_{i, p t}-S_{i, 100}}{S_{i, 100}} \cdot 100 \quad \forall i, p t
$$

An ANOVA is obtained for all previous studied factors, except the factor algorithm (since we are now only studying NA) and adding the factor $p t$. We now summarize the results, from highest to lowest statistical significance indicating the $F$-Ratios and $p$-values between parenthesis: $p t$ factor $(5,996,0.00)$, Number of nodes $(n)(357.3,0.00)$, interaction between $n$ and $p t(122.61,0.00)$, Symmetry (M) $(98.55,0.00)$, interaction between $p t$ and asymmetry $(35.17,0.00)$, interaction between $p t$ and Territory $(\mathrm{T})(31.44,0.00)$, interaction between $p t$ and DemCap (30.63, 0.00), interaction between $p t$ and Location (L) (28.43, 0.00), DemCap (27.05, 0.00), interaction between $p t$ and Depot location (21.04, $0.00)$, Depot location $(20.75,0.00)$, Territory $(15.47,0.00)$ and interaction between Territory and Symmetry $(10.7,0.00)$. As we can see, all studied factors are statistically significant, either in isolation or by means of a two way interaction.

Figure 7 shows the means plot and Tukey's Honest Significant Difference (HSD) confidence intervals of the interaction between $n$ and $p t$.

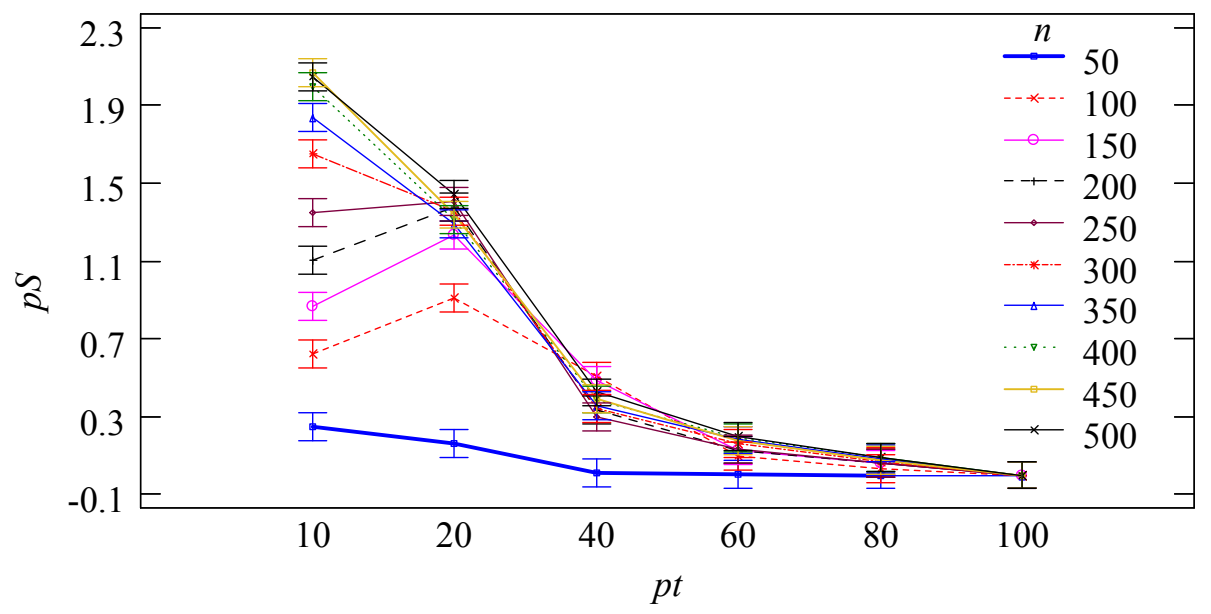

Figure 7: Means plot for $p S$ with Tukey's Honest Significant Difference (HSD) 95\% confidence intervals for the interaction between $n$ and $p t$ factors. NA algorithm.

For lower $p t$ values, NA gives solutions of a much lower quality and this effect is markedly significant for greater $n$ values. The observed deterioration in results is around $2 \%$, in line with the findings of Toth and Vigo (2001), where it is indicated that modern metaheuristics, albeit time consuming, yield results that deviate from optimum solutions between $2 \%$ and $0.5 \%$. It is observed that for small values of $p t$ of $10 \%$ and $20 \%$ the results are worse for $20 \%$ when compared to those of $10 \%$ for some $n$ values. This is due to the 
divergence in the solutions as regards total traveled distance and number of routes. Note that NA was run from scratch each time and it is possible that, in some cases, the result at the first stages of evolution is worse for $p t=20 \%$ than for $p t=10 \%$. In any case, NA is a flexible and robust metaheuristic which adapts very well to asymmetric scenarios. Figure 8 shows the effect of the Symmetry factor.

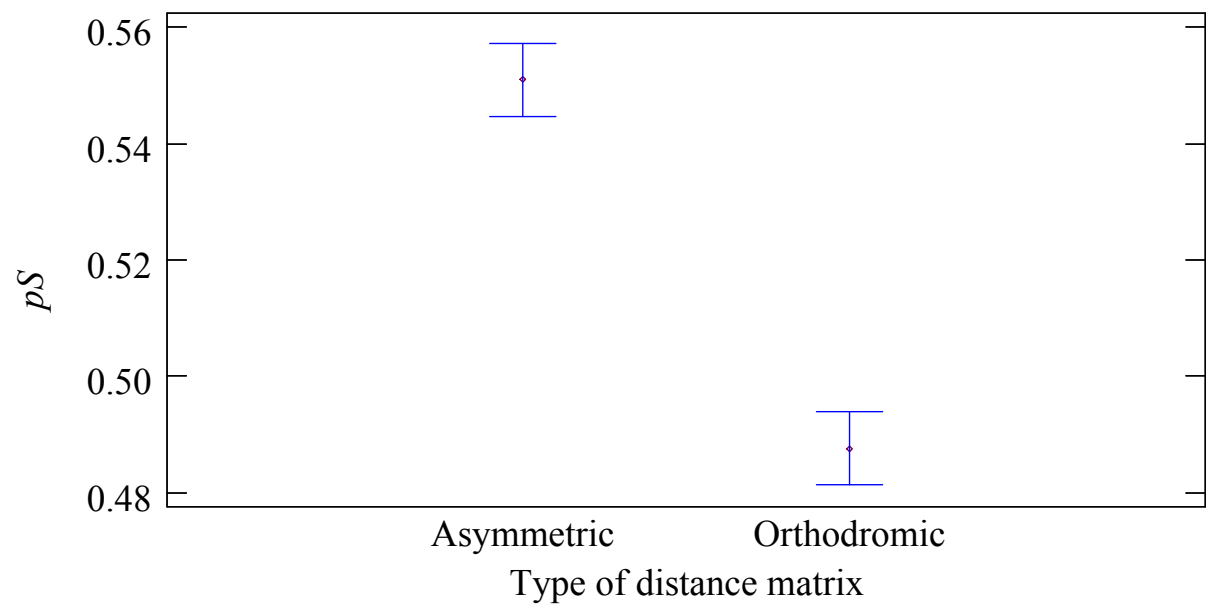

Figure 8: Means plot for $p S$ with Tukey's Honest Significant Difference (HSD) 95\% confidence intervals for the Symmetry (M) factor. NA algorithm.

We see that the level of asymmetry has an effect over the NA method and $p S$ response variable. This effect is small albeit statistically significant. These differences are much greater for $p t=10 \%$ but are non-existent for $p t \geq 40 \%$.

According to Figure 9, the Territory factor also affects NA in a similar way as in the previous experiments. In this new experiment though, the differences are no longer statistically significant when $p t \geq 40 \%$.

We also confirm that the DemCap factor is also affected for smaller processing times $(p t<40 \%)$, shown in Figure 10.

Similarly, Figure 11 shows that the Location factor is affected by the allowed CPU time. Another significant factor is the Depot location depicted in Figure 12. Peripherically located depots pose increased difficulties for NA in short allowed CPU times $(p t \leq 20 \%)$.

To close the study we provide in Table 10 the results of the number of successes $\left(S^{*}\right)$ by the algorithms, number of results (Exp.) and the success rates $\% S^{*}$ according to the type of matrix (Asymmetric as A and Orthodromic as O). For the asymmetric case we see how the success rate of NA decreases by $1.52 \%, 2.53 \%$ for PR and $0.02 \%$ for SW. The algorithm with the highest success rate is NA followed by PR.

Lastly, and according to the several ANOVA experiments carried out, the $\Delta S_{i}^{*}$ indicator is influenced in a statistically significant way by all studied factors. This confirms the 


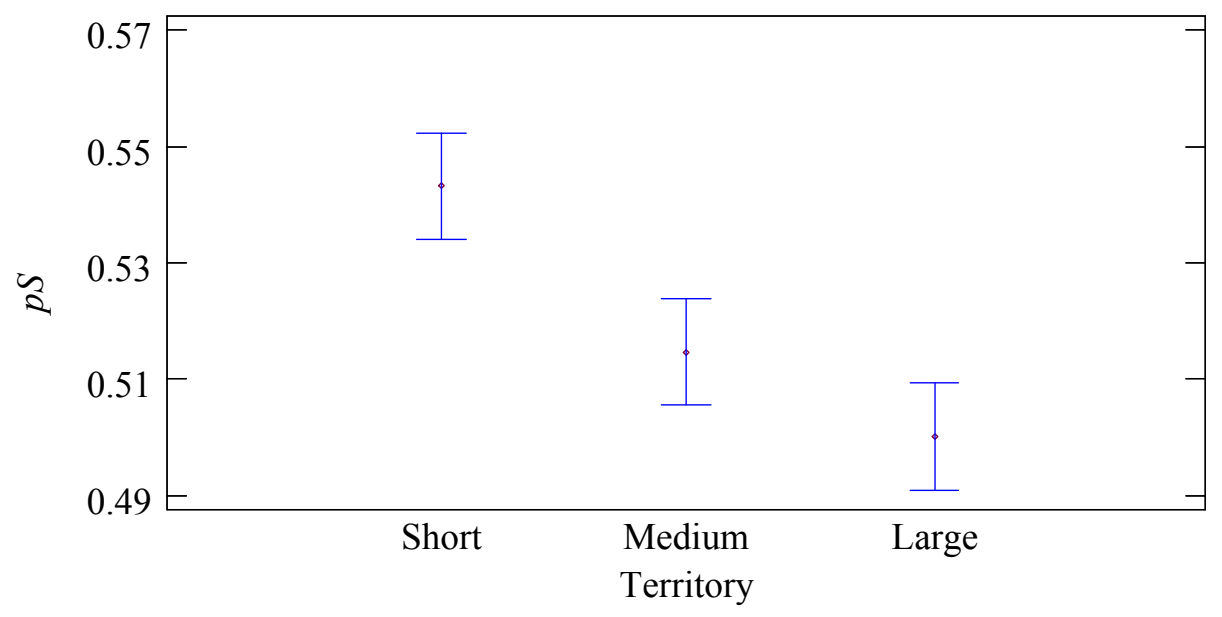

Figure 9: Means plot for $p S$ with Tukey's Honest Significant Difference (HSD) 95\% confidence intervals for the Territory $(\mathrm{T})$ factor. NA algorithm.

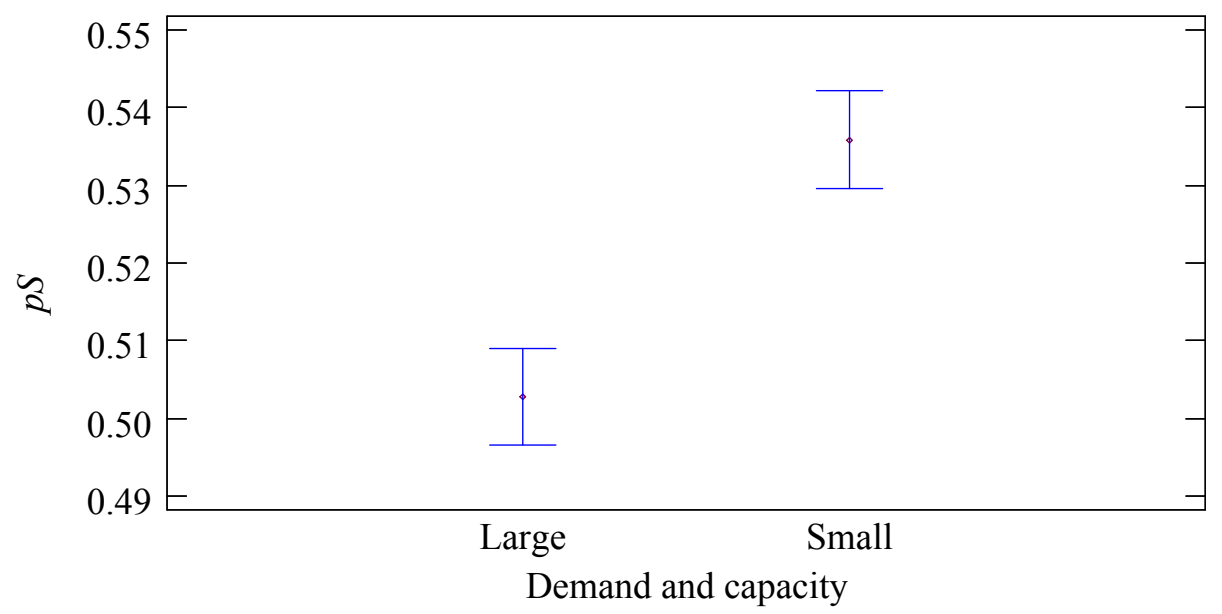

Figure 10: Means plot for $p S$ with Tukey's Honest Significant Difference (HSD) 95\% confidence intervals for the DemCap factor. NA algorithm.

\begin{tabular}{lllllll}
\hline Algorithm & $S^{*}(\mathbf{O})$ & Exp. $(\mathbf{O})$ & $\% S^{*}(\mathbf{O})$ & $S^{*}(\mathbf{A})$ & Exp. $(\mathbf{A})$ & $\% S^{*}(\mathbf{A})$ \\
\hline CW & 0 & 5,400 & $0.00 \%$ & 0 & 5,400 & $0.00 \%$ \\
NA & 10,237 & 27,000 & $37.91 \%$ & 9,827 & 27,000 & $36.40 \%$ \\
PR & 2,874 & 27,000 & $10.64 \%$ & 2,192 & 27,000 & $8.12 \%$ \\
SW & 2 & 5,400 & $0.03 \%$ & 1 & 5,400 & $0.01 \%$ \\
\hline Total & 13,113 & 64,800 & & 12,020 & 64,800 & \\
\hline
\end{tabular}

Table 10: Number of successes $\left(S^{*}\right)$, number of results (Exp.) and the success rates $\% S^{*}$ as a function of the Symmetry factor (Asymmetric as A and Orthodromic as $\mathrm{O}$ ) for the tested methods.

known fact that the orthodromic scenario is more amenable to work with but that all other factors have to be closely observed as well, as they also significatively affect the 


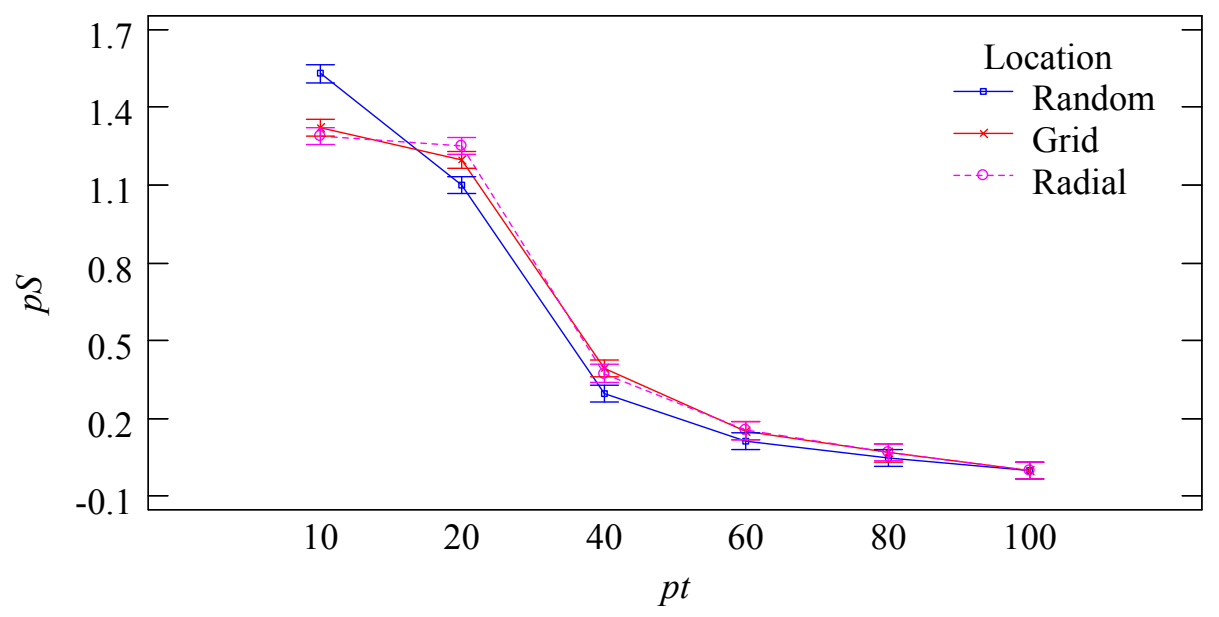

Figure 11: Means plot for $p S$ with Tukey's Honest Significant Difference (HSD) 95\% confidence intervals for the interaction between Location (L) and $p t$ factors. NA algorithm.

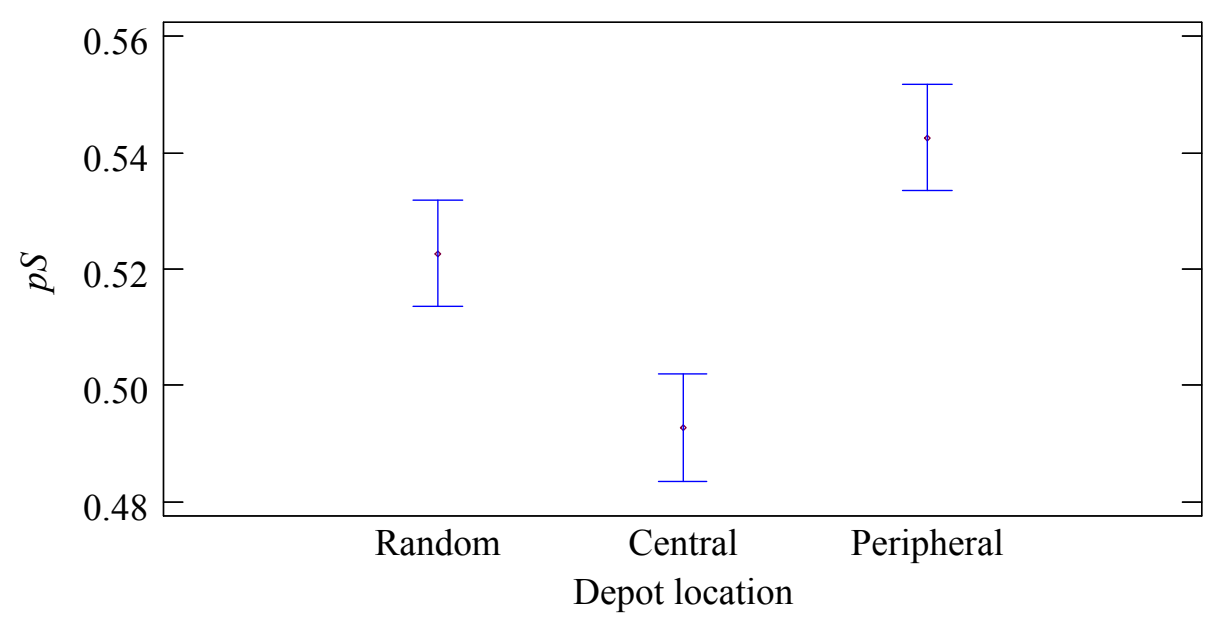

Figure 12: Means plot for $p S$ with Tukey's Honest Significant Difference (HSD) 95\% confidence intervals for the Depot location factor. NA algorithm.

methods. A noteworthy result is the effect that the Depot location factor has over the $\Delta S_{i}^{*}$ response variable, especially for the SW heuristic which is strongly based in the planar properties of the $2 \mathrm{D}$ plane. In this case, the results of the heuristic deteriorate by a large $60 \%$ when the depot is peripherically located with respect to the centrally located depot in the asymmetric scenario.

\section{Conclusions}

In this work we have studied the effect of the asymmetry of transportation networks and other factors over real $C V R P$ instances. We have studied the Territory, Number of nodes $(n)$, Location, Depot location, Asymmetry, client demand and maximum truck capacity as 
factors. All these factors have been examined during thorough computational and statistical experiments. Several heuristic and state-of-the-art metaheuristics have been tested. In a first phase no CPU time limit has been imposed. Later, in a second phase, different CPU time limits have been tested for the NA metaheuristic. Different response variables have been investigated, namely CPU time, quantitative and qualitative comparison of solutions and the quality of solutions, under different indicators.

This paper is a natural extension of the previous study on the much simpler TSP setting (Rodríguez and Ruiz, 2011). The results obtained in the more complex CVRP corroborate previous findings: A higher asymmetry degree in the instances affects in a statistically significant way the CPU time needed by the algorithms and deteriorates the quality of the solutions obtained.

The asymmetry and the number of nodes in a given problem instance are not the only factors affecting the complexity of the $C V R P$. Short distance territories in urban networks and the distribution of the clients in the territory are decisive factors as well. The Depot location is also of paramount importance from a logistics point of view. The demand and truck capacity strongly interact. The result is that routes with a higher number of stops in asymmetric transportation (as in city distribution) challenge routing algorithms.

The main contribution of this work is the demonstration that under the complex reality of the transportation network, the level of asymmetry surfaces and asymmetry have a large effect over the solution methods for the $C V R P$. This effect is many times larger than the outperformance margins between competing state-of-the-art methods when traditional Euclidean distance benchmarks are employed. Given that these results hold true for both the $T S P$ and now for the $C V R P$, it can be inferred that this effect will be present in most derived routing problems. As a final conclusion, we postulate that the scientific community should consider asymmetry and other asymmetry inducing factors when proposing and improving routing algorithms in order for such methods to prove useful in real life environments.

\section{Acknowledgements}

The authors are indebted to Keld Helsgaun, Stefan Røpke and especially to Yuichi Nagata for their kind help, collaboration and for facilitating the binaries of their algorithms. This work is partially funded by the Spanish Ministry of Science and Innovation, under the project "SMPA - Advanced Parallel Multiobjective Sequencing: Practical and Theoretical Advances" with reference DPI2008-03511/DPI. The authors should also thank the IMPIVA - Institute for the Small and Medium Valencian Enterprise, for the project TASER with reference IMDEEA/2011/142. 


\section{References}

Alba, E. (2006). Parallel metaheuristics. A New Class of Algorithms. Wiley, New York.

Christofides, N. and Eilon, S. (1969). Expected distances in distribution problems. Operations Research, 20(4):437-443.

Clarke, G. and Wright, J. (1964). Scheduling of vehicles from central depot to number of delivery points. Operations Research, 12(4):568-581.

Daganzo, C. (1984). The length of tours in zones of different shapes. Transportation Research Part B, 18(2):135-145.

Dubois, N. and Semet, F. (1995). Estimation and determination of shortest-path length in a road network with obstacles. European Journal of Operational Research, 83(1):105116.

Fisher, M. (1994). Optimal solution of vehicle-routing problems using minimum k-trees. Operations Research, 42(4):626-642.

Flood, M. (1956). The TSP. Operations Research, 4(1):61-75.

Gillett, B. and Miller, L. (1974). Heuristic algorithm for vehicle-dispatch problem. Operations Research, 22(2):340-349.

Golden, B., Magnanti, T., and Nguyen, H. (1977). Implementing vehicle routing algorithms. Networks, 7(2):113-148.

Goodchild, M. and Kemp, K. (1990). NCGIA Core Curriculum in GIS. National Center for Geographic Information and Analysis, University of California., Santa Barbara CA.

Helsgaun, K. (2000). An effective implementation of the Lin-Kernighan Traveling Salesman Heuristic. European Journal of Operational Research, 126(1):106-130.

Laporte, G. (2007). What you should know about the vehicle routing problem. Naval Research Logistics, 54(8):811-819.

Laporte, G. (2009). Fifty Years of Vehicle Routing. Transportation Science, 43(4):408-416.

Laporte, G., Mercure, H., and Nobert, Y. (1986). An exact algorithm for the asymmetrical capacitated vehicle-routing problem. Networks, 16(1):33-46.

Love, R. and Morris, J. (1972). Modeling inter-city road distances by mathematical functions. Operational Research Quarterly, 23(1):61-71.

Love, R. and Morris, J. (1988). On estimating road distances by mathematical functions. European Journal of Operational Research, 36(2):251-253.

Nagata, Y. (2007). Edge assembly crossover for the capacitated vehicle routing problem. In Cotta, C. and van Hemert, J. I., editors, Evolutionary Computation in Combinatorial Optimization, 7th European Conference, EvoCOP, Lecture Notes in Computer Science, pages 142-153, Valencia. Springer. 
Pisinger, D. and Røpke, S. (2007). A general heuristic for vehicle routing problems. Computers \& Operations Research, 34(8):2403-2435.

Rodríguez, A. and Ruiz, R. (2011). The effect of the asymmetry of road transportation networks on the traveling salesman problem. In press at Computers $\& 3$ Operations Research, doi:10.1016/j.cor.2011.09.005.

Taillard, É. D. (1993). Parallel iterative search methods for vehicle routing problem. Networks, 23(8):661-693.

Toth, P. and Vigo, D. (2001). An overview of vehicle routing problems. SIAM - Society for Industrial and Applied Mathematics, Philadelphia.

Vincenty, T. (1975). Direct and inverse solutions of geodesics on the ellipsoid with application of nested equations. Survey Review, 23(176):88-93. 